\title{
UM PANORAMA DAS PESQUISAS SOBRE PENSAMENTO COMPUTACIONAL EM PROGRAMAS DE PÓS-GRADUAÇÃO NO BRASIL
}

\author{
João Henrique Berssanette ${ }^{1}$ \\ Antonio Carlos de Francisco²
}

\begin{abstract}
RESUMO
Este artigo apresenta os resultados de uma Revisão Sistemática da Literatura (RSL) que buscou identificar e caracterizar os estudos que tenham como foco o desenvolvimento do pensamento computacional ou que contemplem, em seus objetivos, o estímulo a tal pensamento por meio de práticas educacionais, provenientes de pesquisas de Mestrado e Doutorado produzidas a partir de programas de Pós-Graduação no Brasil, entre os anos de 2010 e 2019. Por meio da execução do protocolo de RSL, foram selecionados 71 estudos para compor a base da revisão, de forma a observar os quantitativos de pesquisas, as UF, instituições e programas de Pós-Graduação, as abordagens e o desenvolvimento, as práticas educacionais, os pressupostos teórico-pedagógicos, os recursos e/ou ferramentas utilizadas, os níveis de ensino, as contribuições e as dificuldades relatadas. Os resultados obtidos retratam o panorama atual das pesquisas sobre pensamento computacional em programas de Pós-Graduação no Brasil, bem como evidenciam o interesse e a atualidade sobre a temática.

Palavras-chave: Pensamento computacional. Programas de Pós-Graduação. Instituições educacionais brasileiras. Revisão Sistemática da Literatura.
\end{abstract}

\section{A PANORAMA OF RESEARCH ON COMPUTATIONAL THINKING IN GRADUATE PROGRAMS IN BRAZIL}

\section{ABSTRACT}

This article presents the results of a Systematic Literature Review (SLR) that sought to identify and characterize studies that focus on the development of computational thinking or that contemplate, in their objectives, the stimulation of such thinking through educational practices, coming from master's and doctoral research produced from graduate programs in Brazil, between the years 2010 and 2019. Through the execution of the SLR protocol, 71 studies were selected to form the basis of the review in order to observe quantitative research, the UF, institutions and graduate programs, approaches and development, educational practices, theoretical-pedagogical assumptions, resources and/or tools used, levels of schooling, contributions and difficulties reported. The results obtained portray the current panorama of research on computational thinking in graduate programs in Brazil, as well as show the interest and actuality on the subject.

Keywords: Computational thinking. Postgraduate programs. Brazilian educational institutions. Systematic literature review.

RECEBIDO EM: 30/11/2020

ACEITO EM: 4/1/2021

\footnotetext{
${ }^{1}$ Autor correspondente. Instituto Federal do Paraná - IFPR - Campus Telêmaco Borba. Telêmaco Borba/PR, Brasil. http://lattes.cnpq. br/4957636385989608. https://orcid.org/0000-0002-7622-3003. joao.berssanette@ifpr.edu.br

2 Universidade Tecnológica Federal do Paraná - UTFPR - Campus Ponta Grossa. Ponta Grossa/PR, Brasil.
} 
Em virtude da relevância dos computadores para a sociedade, tem-se notabilizado o fomento das habilidades relacionadas ao pensamento computacional e à programação de computadores, sendo estas reconhecidas como importantes competências para o século 21 (CHAO, 2016; EINHORN, 2011; GROVER; PEA, 2013; SELBY, 2015; YEN; WU; LIN, 2012).

Em decorrência de tal cenário, diversos países, como Estados Unidos, Reino Unido, Austrália e México, têm promovido educação em ciência da computação (YADAV et al., 2017). Esses esforços destacam a necessidade de preparar os estudantes de hoje para um mundo fortemente influenciado pela computação (SELBY, 2015; YADAV; HONG; STEPHENSON, 2016) e visam, principalmente, a introduzir ideias da ciência da computação, contemplando, assim, o pensamento computacional e a programação de computadores no currículo dos ensinos Fundamental e Médio.

No Brasil, recentemente, a Base Nacional Comum Curricular (BNCC), publicada em 2018, que fundamenta toda a educação básica (BRASIL, 2018), introduz e menciona, por diversas vezes, a expressão pensamento computacional, no entanto o mesmo documento não traz uma definição expressiva do termo, associando-o a uma competência e/ ou habilidade a ser desenvolvida durante o processo de ensino de conteúdos da Matemática (BARBOSA, 2019).

O pensamento computacional tem obtido notório destaque nas discussões sobre educação em computação e a como ensinar tecnologia para estudantes. Assim como na BNCC, todavia, não há um consenso sobre o que é o pensamento computacional entre a comunidade de cientistas da Computação. Embora a expressão pensamento computacional tenha sido cunhado por Papert em 1980, em seu livro Mindstorms: Children, Computers, And Powerful Ideas (PAPERT, 1980), este se popularizou a partir da publicação do artigo "Computational Thinking", de Jeannette Wing (2006).

Wing (2006) define o pensamento computacional como um processo de resolução de problemas, de maneira a projetar sistemas e entender o comportamento humano, com base nos conceitos fundamentais para a Ciência da Computação. Essa autora defende a ideia de que o pensamento computacional é uma habilidade fundamental para todos e não apenas para os cientistas da Computação, devendo ser uma habilidade básica a ser ensinada às crianças, assim como ler, escrever e a aritmética.

Nessa seara, Yadav, Stephenson e Hong (2017) evidenciam que o desenvolvimento do pensamento computacional pelos estudantes permitiria que eles criassem, projetassem e desenvolvessem tecnologias, ferramentas ou sistemas que serão fundamentais no avanço de qualquer campo no futuro.

Desse modo, tem-se observado a instituição de vastas iniciativas, com vistas a se desenvolver o pensamento computacional no contexto da educação, cujo intuito é o de expor os estudantes a conceitos e práticas relacionadas, no sentido de prepará-los para trabalhos em Computação, bem como a pensar fora da caixa e usar habilidades de resolução de problemas com ou sem o apoio de computadores em diferentes áreas de sua vida pessoal, acadêmica e profissional. 
Nesse contexto, a pesquisa sistemática realizada teve como objetivo principal identificar e caracterizar os estudos que tenham como foco o desenvolvimento do pensamento computacional ou que contemplem, em seus objetivos, o estímulo a tal pensamento por meio de práticas educacionais, provenientes de pesquisas de Mestrado e Doutorado produzidas a partir de programas de Pós-Graduação no Brasil.

\section{TRABALHOS RELACIONADOS}

Inicialmente, cumpre destacar que a literatura conta com diversas revisões sobre a temática pensamento computacional no contexto da educação, tanto no âmbito nacional quanto no internacional. Entre essas revisões, algumas têm como foco aspectos gerais do pensamento computacional no contexto educacional, como Zanetti, Borges e Ricarte (2016), Araujo, Andrade e Guerrero (2016), Ortiz e Pereira (2018), Silva, Pereira e Odakura (2018).

Outros estudos têm como foco os desafios e possibilidades do ensino de pensamento computacional nas escolas e a formação de docentes, como Farias, Andrade e Alencar (2015), França e Tedesco (2015), Silva, Silva e França (2017), Hsu, Chang e Hung (2018) e Pasqual Júnior e Oliveira (2019).

Bombasar et al. (2015), Román-González, Moreno-León e Robles (2017), CarvaIho, Netto e Almeida (2017), Zhang e Nouri (2019) e Poulakis e Politis (2020) concentraram seus esforços em identificar as ferramentas e metodologias utilizadas para o ensino do pensamento computacional.

Observam-se, também, estudos que relacionam o pensamento computacional a outras disciplinas, a exemplo da Matemática, como Barcelos et al. (2015) e Barcelos et al. (2018), Hickmott, Prieto-Rodriguez e Holmes (2018). Há, ademais, estudos com foco na forma de avaliação do pensamento computacional, como Araujo, Andrade e Guerrero (2016), Avila et al. (2017) e Tang et al. (2020).

França e Tedesco (2019) traçaram um panorama dos grupos e linhas de pesquisa que investigam o pensamento computacional em diferentes áreas do conhecimento no Brasil. Por fim, outros estudos relacionam o pensamento computacional à programação de computadores e à robótica, como Zanetti, Borges e Ricarte (2016), Buitrago Flórez et al. (2017), Kong, Chiu e Lai (2018), Rodrigues, Aranha e Silva (2018), Agbo et al. (2019), Da Silva e Falcão (2020) e Rosa e Coelho Neto (2020).

Diante do exposto, pondera-se que a revisão sistemática desenvolvida neste artigo difere das mencionadas anteriormente, pois, como base para esta revisão, foram utilizados estudos primários, oriundos de pesquisas de Mestrado e Doutorado desenvolvidas a partir de programas de Pós-Graduação no Brasil.

\section{MÉTODO}

A presente revisão foi desenvolvida com base nas diretrizes para Revisões Sistemáticas de Literatura - RSLs - apresentadas por Kitchenham (2012), tendo como objetivo identificar e caracterizar os estudos primários (teses e dissertações) publicadas entre os anos de 2010 e 2019, provenientes de pesquisas de Mestrado e Doutorado produzidas a partir de programas de Pós-Graduação no Brasil, cujo foco já fora aludido.

O resumo do protocolo desenvolvido para esta revisão é apresentado a seguir na Figura 1. 


\section{Revista \\ \& Educaçãoo}

Figura 1 - Resumo do protocolo RSL desenvolvido

\section{QUESTÕES DE PESQUISA (QP)}

QP1: Quais são os quantitativos de pesquisas produzidas com foco no desenvolvimento do pensamento computacional em programas de Pós-Graduação no Brasil, no período de 2010 a 2019 ?

QP2: Quais são as UF, instituições e programas de Pós-Graduação no Brasil que concentram essas pesquisas?

QP3: Quais são as abordagens desses estudos?

QP4: Como são desenvolvidas essas abordagens?

QP5: Quais são as práticas educacionais associadas ou utilizadas nesses estudos?

QP6: Quais são os pressupostos teórico-pedagógicos associados ou utilizados nesses estudos?

QP7: Quais recursos e/ou ferramentas têm sido utilizados nesses estudos?

QP8: Esses estudos são aplicados em quais níveis de ensino?

QP9: Quais contribuições e/ou dificuldades relacionadas ao pensamento computacional foram relatadas nesses estudos?

\begin{tabular}{|c|c|c|c|}
\hline \multicolumn{4}{|c|}{ PROCESSO DE RECUPERAÇÃO DOS ESTUDOS } \\
\hline$\frac{\text { Expressão de Busca }}{\underline{\text { String }}}$ & "Pensal & "nto Computacional" & \\
\hline$\frac{\text { Fontes de Pesquisa }}{\underline{\text { Databases }}}$ & $\begin{array}{c}\text { Biblioteca Digital de Teses e } \\
\text { Dissertações - BDTD } \\
\mathbf{5 6}\end{array}$ & $\begin{array}{c}\text { Catalogo de Teses e } \\
\text { Dissertações da Capes - CTDC: } \\
91\end{array}$ & $\underline{\text { Total }} \underline{\underline{147}}$ \\
\hline
\end{tabular}

\section{PROCESSO DE SELEÇÃO DOS ESTUdOS}

Remoção dos estudos duplicados $(\mathrm{n}=40)$

Seleção dos estudos com base em critérios de inclusão e exclusão

Critério de Inclusão (CI)

(CI1) Estudos que tenham como foco desenvolvimento do pensamento computacional ou que contemplem, em seus objetivos, o estímulo a tal pensamento por meio de práticas educacionais

\begin{tabular}{|l|}
\hline \multicolumn{1}{|c|}{ Critérios de Exclusão (CE) } \\
\hline $\begin{array}{l}\text { (CE1) Estudos que não abordam as questões de } \\
\text { pesquisa. }\end{array}$ \\
$\begin{array}{l}\text { (CE2) Estudos que se baseiam exclusivamente } \\
\text { em mapeamento ou revisões sistemáticas da } \\
\text { literatura. }\end{array}$ \\
$\begin{array}{l}\text { (CE3) Estudos com acesso indisponível } \\
\text { (divulgação não autorizada). }\end{array}$ \\
\hline
\end{tabular}

\section{AVALIAÇ̃̃o DA QUALIDADE (QA)}

QA1: Existe uma definição clara dos objetivos no estudo?

QA2: Existe uma descrição adequada do contexto em que o estudo foi realizado?

QA3: Existe uma descrição adequada dos métodos de pesquisa empregados no estudo?

QA4: Foi realizado algum tipo de experimento controlado ou estudo de caso para subsidiar os resultados encontrados?

QA5: Existe uma indicação clara dos resultados?

QA6: Os resultados evidenciados representam um contexto educacional real de ensino?

QA7: O estudo tem valor para esta pesquisa?

\section{EXTRAÇ̃̃O E ANÁLISE DE DADOS}

Identificação dos estudos e extração de dados relacionados a questões de pesquisa

Fonte: Desenvolvida pelos autores.

Contexto \& Educação

Editora Unijuí •ISSN 2179-1309 • Ano 36 • no 114 • Maio/Ago. 2021 
Inicialmente com o intuito de obter os dados necessários para responder às questões de pesquisa, procedeu-se à recuperação dos estudos, com base nos termos que compõem o escopo desta revisão (pensamento computacional), sendo utilizada a seguinte expressão de busca (string): "Pensamento Computacional".

Dessa forma, essa expressão de busca foi instituída de forma automática por meio dos motores de busca, tendo como parâmetros os campos título (title), palavras-chave (keywords) e resumo (abstract), além de documentos do tipo tese ou dissertação, publicados entre os anos de 2010 e 2019. A busca foi realizada em 21 de setembro de 2020 e identificou, nas bases de dados destacadas, um total de 147 estudos.

A partir da recuperação dos estudos, iniciou-se o processo de seleção para compor o corpus documental para revisão. Assim, para esta revisão, inicialmente, foram descartados os estudos duplicados $(n=40)$. Em seguida, os 107 restantes foram submetidos a uma análise dos metadados título (title), palavras-chave (keywords) e resumo (abstract) pelos pesquisadores de forma individual, observando os critérios de inclusão e exclusão.

Conforme já mencionado, os dois pesquisadores realizaram o processo de seleção de forma independente para cada um dos estudos. Os motivos para inclusão ou exclusão dos estudos foram cuidadosamente registrados e, em seguida, foram realizadas reuniões para se discutir e resolver as divergências que resultaram dessa análise.

$\mathrm{Na}$ sequência, considerando que a avaliação da qualidade dos estudos é uma preocupação presente entre as diretrizes que disciplinam a revisão sistemática, pois, por meio dela, pode-se identificar e desconsiderar estudos metodologicamente frágeis, com resultados incipientes ou de baixa qualidade científica (GALVÃO; PEREIRA, 2015), procedeu-se à avaliação da qualidade dos estudos pré-selecionados a partir dos critérios estabelecidos.

Para avaliar a qualidade dos estudos que compõem esta revisão, foram desenvolvidas sete questões, com base em uma adaptação das questões propostas por Dybå e Dings $\varnothing$ yr (2008). Para responder a essas questões foi realizada uma leitura minuciosa dos estudos pré-selecionados com base nos critérios estabelecidos. As questões foram ponderadas da seguinte forma: 1 (sim, o estudo selecionado satisfaz o critério); 0.5 (parcialmente, o estudo selecionado não relata claramente) e 0 (não, o estudo selecionado não satisfaz o critério). Ao final da avaliação foi realizada a somatória das pontuações obtidas pelo estudo com base nas questões de avaliação.

As questões de avaliação da qualidade formuladas visam a identificar a relevância do estudo no que diz respeito aos seus objetivos, metodologia e resultados, bem como sua confiabilidade e valor para esta revisão. Dessa forma, como regra de corte para que os estudos viessem a compor a base para esta revisão, definiram-se estudos que obtivessem pontuação superior ou igual a 4.

O procedimento realizado para a avaliação da qualidade dos estudos foi similar ao processo de seleção, ou seja, cada pesquisador produziu uma avaliação independente dos estudos. Ao término do processo foram organizadas reuniões para discutir e resolver as divergências que resultaram desse processo de avaliação. 
Por fim, foi realizada a extração e análise dos dados. Nessa fase, os estudos selecionados para compor o corpus documental desta revisão foram identificados e disponibilizados em uma planilha compartilhada, em que um pesquisador procedeu à extração dos dados relacionados às questões de pesquisa, com o outro verificando essa extração. É pertinente ressaltar que quaisquer divergências eram discutidas até que a situação fosse resolvida.

A partir dessa extração os dados foram sintetizados e agrupados em categorias, possibilitando, assim, a análise que propiciou caracterizar os estudos selecionados.

\section{APLICAÇÃO DO PROTOCOLO, RESULTADOS E ANÁLISES}

A busca por estudos para esta revisão seguiu o processo descrito na seção anterior. Assim como já mencionado, o processo de recuperação de estudos, de forma automática e por meio dos motores de busca nas bases científicas elencadas, retornou, inicialmente, um total de 147 estudos.

Os estudos recuperados foram submetidos a um processo de análise de registros duplicados. Nesse processo foram descartados 40 estudos duplicados, restando, assim, 107 trabalhos, os quais, durante o processo de seleção, foram submetidos a uma análise dos metadados título (title), palavras-chave (keywords) e resumo (abstract), conforme os critérios de inclusão e exclusão definidos.

Assim, a partir do processo de seleção, dos 107 estudos recuperados 36 foram descartados, ou por não atenderam ao critério de inclusão, ou por serem classificados em algum dos critérios de exclusão, o que resultou em 71 estudos pré-selecionados.

Os estudos pré-selecionados foram, então, avaliados quanto à sua qualidade. No que se refere a essa avaliação, cabe destacar que a maioria dos estudos pré-selecionados recebeu uma boa pontuação, obtendo, em média, seis pontos, o que era, até certo modo, esperado, tendo em vista que esses estudos são oriundos do desenvolvimento de pesquisas de Mestrado e Doutorado submetidas a bancas de avaliação.

Dessa forma, no que respeita à qualidade dos estudos pré-selecionados, nenhum deles obteve pontuação inferior a 4 pontos; desse modo, nenhum deles foi descartado com base na avaliação da qualidade desenvolvida. Nesse sentido, compõem a base para esta revisão 9 teses e 62 dissertações, totalizando 71 estudos selecionados. Tais estudos podem ser averiguados no Apêndice A - Estudos Selecionados. Além disso, todos os dados relativos à pesquisa podem ser consultados por meio do seguinte link: bit.ly/ Artigo-PC2021-RCE.

Por fim, compete registrar que, durante todo o processo de execução do protocolo da RSL em questão, houve um bom acordo entre os pesquisadores, sendo registrados, tanto no processo de seleção quanto no processo de avaliação dos estudos, índices de divergências inferiores a 5\%.

Desse modo, a seguir, são apresentadas as respostas para as questões de pesquisa estipuladas; nelas, são evidenciados os resultados mais representativos, bem como as análises, a partir do panorama traçado, que poderão nortear futuras investigações sobre o tema no Brasil. 
Diante do exposto, entretanto, cumpre registrar que os resultados menos expressivos, os quais se fazem presentes na amostra, podem ser consultados por meio do acesso aos dados da pesquisa já disponibilizados.

\section{Quantitativos de Pesquisas Produzidas (QP1)}

Por meio dos estudos que compõem a base desta revisão, observou-se que os quantitativos de pesquisas com foco no pensamento computacional, desenvolvidas em programas de Pós-Graduação no Brasil (Gráfico 1), dentro do período pesquisado, registram uma lacuna entre os anos de 2010 e 2012, observando-se que o primeiro registro de pesquisa aparece em 2013, que conta com apenas uma pesquisa. Os anos de 2014 e 2015, por sua vez, contam com três pesquisas cada. A partir do ano de 2016 observa-se uma tendência de crescimento de trabalhos, dado que o referido ano conta com 8 pesquisas desenvolvidas, 2017 com 16, 2018 com 18 e 2019 com 22.

Gráfico 1-Quantitativos de pesquisas produzidas

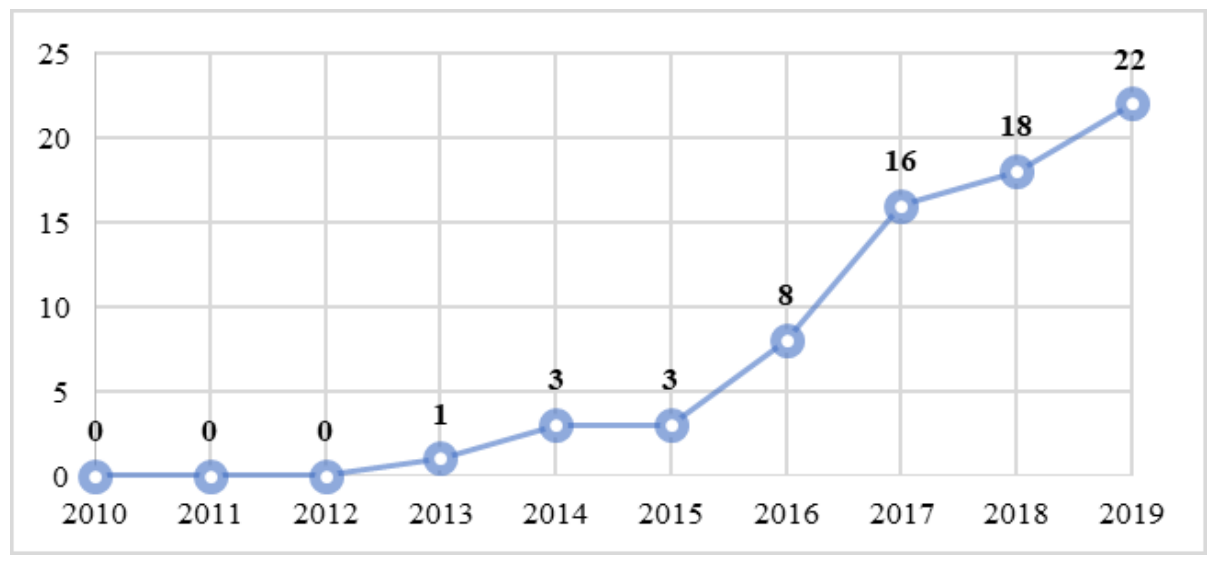

Fonte: Desenvolvido pelos autores.

Esses quantitativos evidenciam o interesse e a atualidade da temática, bem como a relevância e a notabilidade do fomento ao desenvolvimento das habilidades relacionadas ao pensamento computacional, indicando, ainda, que se trata de um campo com potencial promissor para o desenvolvimento de pesquisas. Salienta-se que tal indicação está em consonância com estudos como Ortiz e Pereira (2018), Silva, Pereira e Odakura (2018), Carvalho, Netto e Almeida (2017), Araujo, Andrade e Guerrero (2016) e Zanetti, Borges e Ricarte (2016).

\section{Unidades da Federação (UF), instituições e programas de Pós-Graduação que concentram essas pesquisas (QP2)}

No que se refere às Unidades da Federação, que concentram os estudos com foco no pensamento computacional (Gráfico 2), as pesquisas estão distribuídas nas seguintes UFs: SP, com 15 estudos; RS, com 14; SC, com 10; PR, com 8; PB, com 5; RJ, com 4; $B A$, com 3; AM, DF, PE, RN e SE, com 2 estudos cada; CE e PA, com 1 estudo cada. 


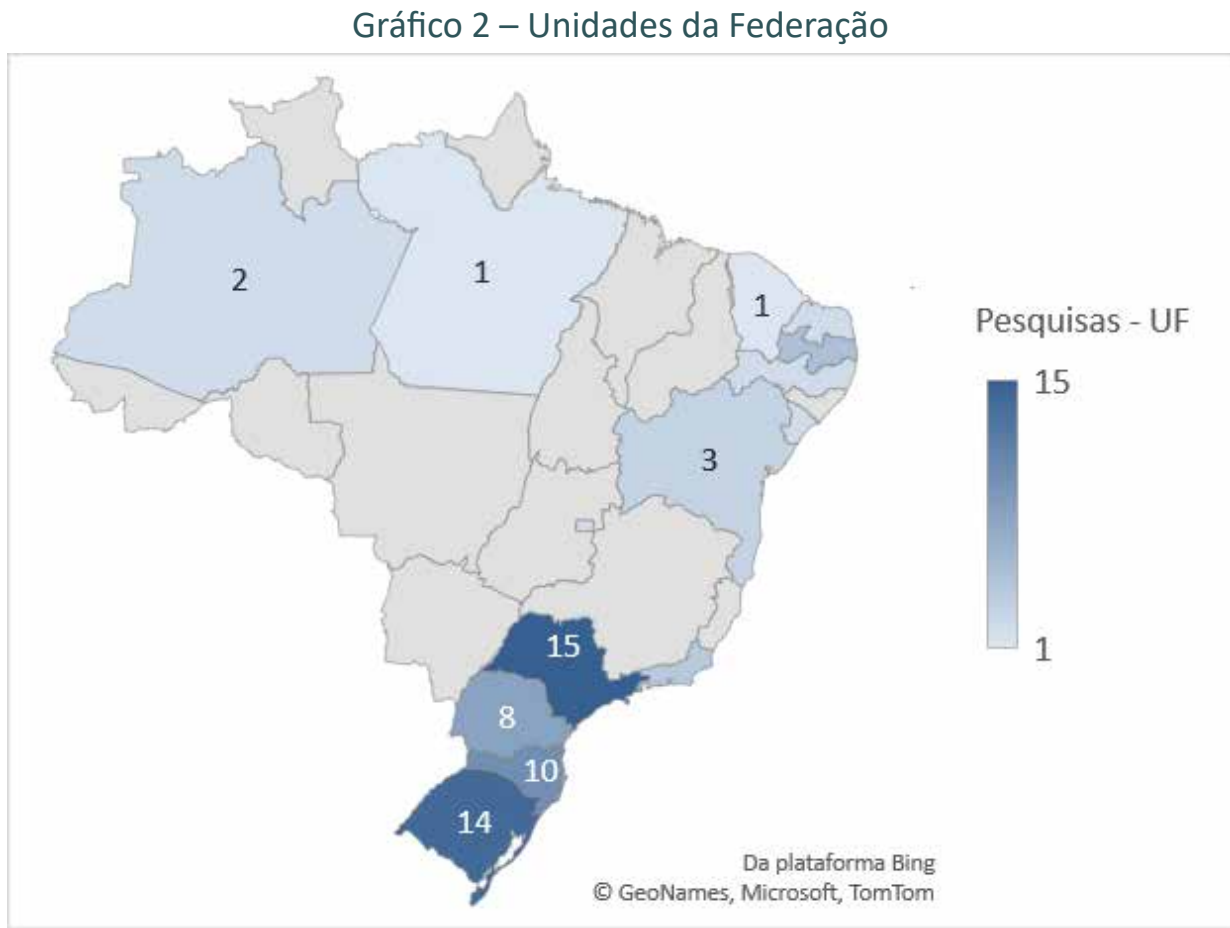

Fonte: Desenvolvido pelos autores.

Ainda no que se refere às Unidades da Federação, que concentram os estudos com foco no pensamento computacional (Gráfico 2), cabe destacar que os achados da pesquisa estão relativamente em conformidade com os observados por Silva, Pereira e Odakura (2018), bem como a distribuição geográfica de grupos de pesquisa sobre PC, averiguada por França e Tedesco (2019), de modo a evidenciar a concentração desses grupos, principalmente nas regiões Sudeste, Sul e Nordeste.

Em relação às instituições onde essas pesquisas foram desenvolvidas, verificamos, na Tabela 1, a quantidade de trabalhos produzidos nessas instituições, bem como o respectivo percentual no que respeita à amostra de estudos selecionados.

Tabela 1 - Instituições e quantitativos de pesquisas

\begin{tabular}{lcc}
\multicolumn{1}{c}{ INSTITUIÇÕES } & QUANTIDADE & \% \\
\hline Universidade do Vale do Itajaí & 8 & $11,27 \%$ \\
\hline Universidade Federal de Campina Grande & 5 & $7,04 \%$ \\
\hline Universidade Estadual de Campinas & 3 & $4,23 \%$ \\
\hline Universidade Estadual Paulista & 3 & $4,23 \%$ \\
\hline Universidade Federal do Rio Grande do Sul & 3 & $4,23 \%$ \\
\hline
\end{tabular}

Fonte: Desenvolvida pelos autores.

Como pode ser observado na Tabela 1 , em relação às instituições onde as pesquisas foram desenvolvidas, merecem destaque as seguintes: Universidade do Vale do Itajaí, com 8 pesquisas; Universidade Federal de Campina Grande, com 5; Universidade Estadual de Campinas, Universidade Estadual Paulista e Universidade Federal do Rio Grande do Sul, com 3 pesquisas cada.

Referente aos programas de Pós-Graduação nos quais essas pesquisas foram produzidas, a seguir verificamos os dados na Tabela 2. 
Tabela 2 - Programas de Pós-Graduação e quantitativos de pesquisas

\begin{tabular}{lcc}
\hline \multicolumn{1}{c}{ PROGRAMAS DE PÓS-GRADUAÇão } & QUANTIDADE & $\%$ \\
\hline Ciência da Computação & 14 & $19,72 \%$ \\
\hline Computação Aplicada & 8 & $11,27 \%$ \\
\hline Educação & 6 & $8,45 \%$ \\
\hline Matemática & 5 & $7,04 \%$ \\
\hline Informática & 4 & $5,63 \%$ \\
\hline Informática na Educação & 4 & $5,63 \%$ \\
\hline
\end{tabular}

Fonte: Desenvolvida pelos autores.

Ao ter em vista a Tabela 2, destacam-se os seguintes dados: Programa de Pós-Graduação em Ciência da Computação, com 14 pesquisas; Computação Aplicada, com 8; Educação, com 6; Matemática, com 5; Informática e Informática na Educação, com 4 pesquisas cada.

Ainda no que se refere aos programas de Pós-Graduação, observa-se que os resultados estão relativamente em conformidade com os observados por França e Tedesco (2019) quanto ao domínio de conhecimento dos grupos de pesquisa sobre pensamento computacional.

\section{Abordagens dos estudos (QP3)}

Em relação às abordagens dos estudos que têm como foco o desenvolvimento do pensamento computacional (PC), na Tabela 3 são identificados os estudos dentro de cada uma dessas abordagens, bem como são destacados o quantitativo e o percentual no que concerne à amostra.

Tabela 3 - Abordagens dos estudos

\begin{tabular}{|c|c|c|c|}
\hline ABORDAGEM & ESTUDO & QTD. & $\%$ \\
\hline & $\begin{array}{l}\text { E01; E06; E08; E09; E10; E11; E13; E20; E24; } \\
\text { E25; E26; E27; E32;E37; E38; E39;E44; E46; } \\
\text { E51;E53;E54;E55;E56;E57; E60;E61;E66; } \\
\text { E67.E71 }\end{array}$ & & \\
\hline Promoção do PC & E67; E71 & 29 & $40,85 \%$ \\
\hline $\begin{array}{l}\text { Associação do PC } \\
\text { à Programação de } \\
\text { Computadores }\end{array}$ & $\begin{array}{l}\text { E01; E02; E04; E07; E12; E15; E21; E22; E25; } \\
\text { E27; E29; E35; E43; E46; E47; E48; E49; E60; } \\
\text { E66; E67; E68; E69 }\end{array}$ & 22 & $30,99 \%$ \\
\hline $\begin{array}{l}\text { Associação do PC à } \\
\text { Matemática }\end{array}$ & $\begin{array}{l}\text { E05; E07; E14; E15; E17; E21; E23; E24; E29; } \\
\text { E34; E40;E42; E50; E52;E57; E58; E59; E62 }\end{array}$ & 18 & $25,35 \%$ \\
\hline Avaliação do PC & $\begin{array}{l}\text { E02; E03; E14; E18; E20; E22; E28; E30; E36; } \\
\text { E45; E64; E70 }\end{array}$ & 12 & $16,90 \%$ \\
\hline Associação do PC à Robótica & E06; E16; E32; E49; E56; E59; E63; E65; E69 & 9 & $12,68 \%$ \\
\hline $\begin{array}{l}\text { Associação do PC à Língua } \\
\text { Portuguesa }\end{array}$ & E31; E41; E57 & 3 & $4,23 \%$ \\
\hline
\end{tabular}

Fonte: Desenvolvida pelos autores.

Como pode ser observado na Tabela 3, 40,85\% ( $n=29)$ dos estudos têm como abordagem a promoção do pensamento computacional; 30,99\% ( $n=22)$ abordam o PC associado à programação de computadores; $25,35 \%(n=18)$ abordam o PC associado à Matemática; $16,90 \%(n=12)$ têm como abordagem a avaliação do PC; $12,68 \%(n=9)$ 
abordam o PC associado à robótica; 4,23\% $(n=3)$ abordam o PC associado à Língua Portuguesa. Além disso, vale registrar que alguns estudos utilizam-se de mais de uma abordagem; desse modo, os trabalhos foram classificados em ambas as abordagens.

Os resultados relativos às abordagens dos estudos que compõem a base para esta revisão estão moderadamente em consonância com as pesquisas de Araujo, Andrade e Guerrero (2016).

\section{Desenvolvimento das abordagens (QP4)}

No que diz respeito ao desenvolvimento dessas abordagens, na Tabela 4 são identificados os estudos dentro das propostas de desenvolvimento, bem como são destacados o quantitativo e o percentual em relação à amostra.

Tabela 4 - Desenvolvimento das abordagens nos estudos

\begin{tabular}{|c|c|c|c|}
\hline DESENVOLVIMENTO & ESTUDO & QTD. & $\%$ \\
\hline $\begin{array}{l}\text { Efetivação de intervenção } \\
\text { pedagógica ou cursos/projetos } \\
\text { de extensão (oficinas, minicursos, } \\
\text { MOOCs, workshops) }\end{array}$ & $\begin{array}{l}\text { E01; E04; E07; E08; E10; E11; E13; E14; } \\
\text { E15; E16; E17; E19; E21; E22; E23; E24; } \\
\text { E25; E29; E31;E33; E34; E35; E36; E39; } \\
\text { E41;E44; E47;E48; E50; E56; E57; E58; } \\
\text { E59;E60; E63;E64; E65; E66; E67; E69; } \\
\text { E70 }\end{array}$ & 41 & $57,75 \%$ \\
\hline $\begin{array}{l}\text { Desenvolvimento de ferramentas } \\
\text { ou instrumentos }\end{array}$ & $\begin{array}{l}\text { E01; E06; E09; E14; E26; E27; E35; E38; } \\
\text { E40; E46; E49; E51;E54; E55; E61;E64; } \\
\text { E71 }\end{array}$ & 17 & $23,94 \%$ \\
\hline $\begin{array}{l}\text { Desenvolvimento de proposta } \\
\text { de abordagem (estratégia } \\
\text { pedagógica, metodologia ou } \\
\text { modelo) }\end{array}$ & $\begin{array}{l}\text { E05; E12; E25; E27; E37; E39; E42; E43; } \\
\text { E44; E68 }\end{array}$ & 10 & $14,08 \%$ \\
\hline $\begin{array}{l}\text { Análise ou avaliação de } \\
\text { desempenho ou percepção dos } \\
\text { estudantes }\end{array}$ & E18; E20; E52 & 3 & $4,23 \%$ \\
\hline $\begin{array}{l}\text { Desenvolvimento de instrumentos } \\
\text { ou modelos de avaliação }\end{array}$ & E02; E03; E30 & 3 & $4,23 \%$ \\
\hline
\end{tabular}

Fonte: Desenvolvida pelos autores.

Como pode ser notado na Tabela 4, o desenvolvimento da abordagem por meio da instituição de intervenção pedagógica ou cursos/projetos de extensão (oficinas, minicursos, MOOCs, workshops) foi observado em $57,75 \%(n=41)$ dos estudos.

Nesse sentido, cumpre destacar que o uso de intervenções pedagógicas ou experimentos, para avaliar as diferentes práticas educacionais, parece adequado para se coletar evidências empíricas sobre a sua efetividade, bem como as eventuais contribuições e suas limitações de instauração.

Além disso, merece destaque o desenvolvimento da abordagem por meio do desenvolvimento de ferramentas ou instrumentos, observado em $23,94 \%$ ( $n=17)$ dos estudos, e o desenvolvimento de proposta de abordagem (estratégia pedagógica, metodologia ou modelo), observado em $14,08 \%(n=10)$ dos estudos. 


\section{Práticas educacionais associadas ou utilizadas (QP5)}

No que diz respeito às práticas educacionais associadas ou utilizadas nesses trabalhos, na Tabela 5 são identificados os estudos e são caracterizadas essas práticas, bem como são destacados o quantitativo e o percentual em relação à amostra.

Tabela 5 - Práticas educacionais associadas ou utilizadas nos estudos

\begin{tabular}{|c|c|c|c|}
\hline PRÁTICAS EDUCACIONAIS & ESTUDO & QTD. & $\%$ \\
\hline Indeterminado & $\begin{array}{l}\text { E01; E02; E03; E06; E14; E18; E20; } \\
\text { E22; E25; E28; E30; E32; E38; E41; } \\
\text { E44; E45; E47; E50;E54; E58; E59; } \\
\text { E62;E65;E66;E68 }\end{array}$ & 25 & $35,21 \%$ \\
\hline $\begin{array}{l}\text { Desenvolvimento ou utilização de } \\
\text { jogos (digitais ou analógicos) }\end{array}$ & $\begin{array}{l}\text { E04; E07; E09; E10; E21; E34; E39; } \\
\text { E48; E55; E60; E61; E64 }\end{array}$ & 12 & $16,90 \%$ \\
\hline Resolução de problemas & $\begin{array}{l}\text { E13; E15; E17; E27; E31; E36; E40; } \\
\text { E42; E43; E48; E52; E55 }\end{array}$ & 12 & $16,90 \%$ \\
\hline $\begin{array}{l}\text { Aprendizagem baseada em } \\
\text { atividades práticas }\end{array}$ & $\begin{array}{l}\text { E08; E16; E17; E19; E23; E31; E49; } \\
\text { E56; E60; E70 }\end{array}$ & 10 & $14,08 \%$ \\
\hline Atividades desplugadas & $\begin{array}{l}\text { E11; E12; E23; E24; E29; E36; E37; } \\
\text { E39; E57 }\end{array}$ & 9 & $12,68 \%$ \\
\hline Atividades lúdicas & E29; E31; E35; E39; E49; E53; E67 & 7 & $9,86 \%$ \\
\hline $\begin{array}{l}\text { Aprendizagem baseada em } \\
\text { problemas }\end{array}$ & E07; E15; E37; E64; E69 & 5 & $7,04 \%$ \\
\hline Atividades plugadas & E12; E23; E36; E37; E39 & 5 & $7,04 \%$ \\
\hline Aprendizagem baseada em jogos & E26; E46; E51; E71 & 4 & $5,63 \%$ \\
\hline $\begin{array}{l}\text { Aprendizagem baseada em } \\
\text { projetos }\end{array}$ & E04; E13; E57; E63 & 4 & $5,63 \%$ \\
\hline Aprendizagem colaborativa & E05; E27; E33 & 3 & $4,23 \%$ \\
\hline
\end{tabular}

Conforme pode ser verificado na Tabela 5, em 35,21\% ( $n=25)$ dos estudos não se faz possível determinar quais foram as práticas educacionais associadas ou utilizadas. Entre as práticas observadas, merecem destaque: o desenvolvimento ou utilização de jogos (digitais ou analógicos) e a resolução de problemas, observados em $16,90 \%$ $(n=12)$ dos estudos cada; aprendizagem baseada em atividades práticas (Hand's On/ mão na massa), em 14,08\% ( $n=10$ ); atividades desplugadas, em $12,68 \%$ ( $n=9$ ) e atividades lúdicas, em 9,86\% ( $n=7)$.

Ainda no que se refere às práticas educacionais, convém registrar que as práticas: desenvolvimento ou utilização de jogos, aprendizagem baseada em atividades práticas e atividades desplugadas também foram observadas por Silva, Pereira e Odakura (2018).

\section{Pressupostos teórico-pedagógicos associados ou utilizados (QP6)}

Em relação aos pressupostos teórico-pedagógicos associados ou utilizados nos estudos (Tabela 6), o construcionismo, teoria proposta por Seymour Papert, é observado em $28,17 \%(n=20)$ dos estudos. Já o construtivismo, geralmente associado a Piaget, mas não exclusivamente a ele, é observado em 14,08\% ( $n=10)$. 
Além deles, destacam-se: a teoria da aprendizagem significativa, proposta por Ausubel, e a teoria sociointeracionista de Vygotsky, ambas observadas em 5,63\% ( $n=4)$ dos estudos cada. Também foi averiguado o Método de Polya em 4,23\% ( $n=3)$ dos estudos. Importa registrar que em $52,11 \%$ ( $n=37)$ não foi possível determinar a recorrência dos pressupostos teórico-pedagógicos utilizados.

Tabela 6 - Pressupostos teórico-pedagógicos associados ou utilizados nos estudos

\begin{tabular}{llcc}
\hline $\begin{array}{c}\text { PRESSUPOSTOS } \\
\text { TEÓRICO-PEDAGÓGICOS }\end{array}$ & \multirow{2}{*}{ ESTUDO } & \multirow{2}{*}{$\%$} \\
\hline & E01; E02; E03; E04; E05; E06; E09; E11; E12; E14; & \\
& E15; E18; E20; E23; E24; E27; E28; E30; E32; E35; & \\
& E36; E38; E39; E40; E41; E42; E43; E46; E47; E51; & \\
Indeterminado & E52; E57; E59; E61; E65; E69; E71 & 37 & $52,11 \%$ \\
\hline & E07; E13; E16; E19; E21; E22; E25; E26; E29; E34; & \\
Construcionismo & E45; E50; E53; E54; E55; E58; E63; E64; E67; E70 & 20 & $28,17 \%$ \\
\hline Construtivismo & E13; E16; E21; E29; E31; E45; E49; E56; E67; E68 & 10 & $14,08 \%$ \\
\hline $\begin{array}{l}\text { Aprendizagem } \\
\text { significativa }\end{array}$ & E10; E33; E50; E68 & 4 & $5,63 \%$ \\
\hline Teoria sociointeracionista & E13; E48; E53; E60 & 4 & $5,63 \%$ \\
\hline Método de Polya & E13; E17; E29 & 3 & $4,23 \%$ \\
\hline
\end{tabular}

Fonte: Desenvolvida pelos autores.

Ainda no que se refere a esse questionamento, cabe destacar que os resultados observados estão parcialmente de acordo com os apresentados por Silva, Pereira e Odakura (2018).

\section{Recursos e/ou ferramentas utilizados (QP7)}

Referente aos recursos e/ou ferramentas utilizados, na Tabela 7 são identificados os estudos, bem como são destacados o quantitativo e o percentual em relação à amostra.

Tabela 7 - Recursos e/ou ferramentas utilizados nos estudos

\begin{tabular}{llcc}
\hline $\begin{array}{c}\text { RECURSOS E/OU } \\
\text { FERRAMENTAS }\end{array}$ & \multicolumn{1}{c}{ ESTUDO } & QTD. & \% \\
\hline \multirow{3}{*}{ Indeterminado } & $\begin{array}{l}\text { E01; E03; E09; E11; E17; E20; E27; E28; E29; E30; } \\
\text { E31; E36; E40; E41; E44; E51; E52; E62; E68; E71 }\end{array}$ & 20 & $28,17 \%$ \\
\hline & E04; E07; E13; E15; E21; E22; E23; E24; E33; E34; & & \\
Scratch & E35; E37; E39; E48; E50; E58; E60; E64; E66; E67 & 20 & $28,17 \%$ \\
\hline Robótica educacional & E06; E16; E32; E49; E56; E59; E63; E65 & 8 & $11,27 \%$ \\
\hline Code.org/Hora do Código & E04; E14; E37; E39; E45; E66 & 6 & $8,45 \%$ \\
\hline Arduino & E16; E19; E37; E49; E59 & 5 & $7,04 \%$ \\
\hline
\end{tabular}

Fonte: Desenvolvida pelos autores.

Como pode ser observado na Tabela 7, merecem destaque os seguintes recursos e/ou ferramentas: Scratch, presente em 28,17\% ( $n=20)$ dos estudos; robótica educacional, em 11,27\% ( $n=8)$; Code.org/Hora do Código (Hour Of Code), em 8,45\% ( $n=6)$, e Arduino, em $7,04 \%(n=5)$. Cabe registrar que em $28,17 \%(n=20)$ dos estudos não foi possível determinar a recorrência dos recursos e ferramentas utilizados. 
Ainda no que se refere aos recursos e/ou ferramentas, vale destacar que a ferramenta Scratch e a robótica educacional (Kits) estão em consonância com os resultados observados por Ortiz e Pereira (2018), bem como Carvalho, Netto e Almeida (2017), que adicionam Code.org/Hora do Código aos listados anteriormente.

\section{Níveis de ensino onde são aplicados (QP8)}

No que se refere à aplicação dos estudos (Tabela 8), observa-se que $77,46 \%$ $(n=55)$ dos estudos selecionados para comporem a base desta revisão foram, de algum modo, aplicados. Merece destaque, todavia, o fato de que a maior parcela dos estudos - 42,25\% ( $n=30)$ - concentra-se no Ensino Fundamental, seguido pelo Ensino Médio, com $21,13 \%(n=15)$.

Tabela 8 - Aplicação e níveis de ensino dos estudos

\begin{tabular}{|c|c|c|c|}
\hline NÍVEL DE ENSINO & ESTUDO & QTD. & $\%$ \\
\hline \multirow[b]{4}{*}{ Ensino Fundamental } & E06; E09; E10; E11; E13; E14; E15; E17; E21; & & \\
\hline & E23; E25; E26; E29; E31; E34; E35; E36; E39; & & \\
\hline & E41; E46; E47; E49; E50; E56; E57; E58; E59; & & \\
\hline & $\mathrm{E} 60 ; \mathrm{E} 63 ; \mathrm{E} 67$ & 30 & $42,25 \%$ \\
\hline \multirow[b]{2}{*}{ Não aplicado } & E01; E02; E03; E18; E20; E28; E32; E38; E40; & & \\
\hline & E42; E43; E45; E61; E62; E68; E71 & 16 & $22,54 \%$ \\
\hline & E06; E09; E13; E16; E25; E27; E33; E36; E41; & & \\
\hline Ensino Médio & E46; E48; E52; E65; E69; E70 & 15 & $21,13 \%$ \\
\hline Ensino Superior & E05; E07; E19; E30; E52; E55; E64 & 7 & $9,86 \%$ \\
\hline Ensino Técnico & E04; E07; E08; E33; E64; E66 & 6 & $8,45 \%$ \\
\hline Educação Infantil & E12; E51; E53; E56 & 4 & $5,63 \%$ \\
\hline \multicolumn{4}{|l|}{ Formação de } \\
\hline Professores & E24; E31; E37; E41 & 4 & $5,63 \%$ \\
\hline
\end{tabular}

Fonte: Desenvolvida pelos autores.

Ainda no que diz respeito aos níveis de ensino, em que são aplicados os estudos sobre pensamento computacional, cabe destacar que os achados desta pesquisa coadunam-se aos de Silva, Pereira e Odakura (2018) e Rodrigues, Aranha e Silva (2018) em âmbito nacional, bem como Ortiz e Pereira (2018) que, ao realizarem um mapeamento de iniciativas para promover o pensamento computacional em escala global, observaram que a educação básica (Ensinos Fundamental e Médio) concentra a maior parte dessas iniciativas.

\section{Contribuições e/ou dificuldades relatadas (QP9)}

No que se refere às contribuições relacionadas ao pensamento computacional, relatadas pelos estudos que compõem a base desta revisão, na Tabela 9 são sistematizadas essas contribuições, bem como são identificados os estudos, destacando-se o quantitativo e o percentual em relação à amostra. 
Tabela 9 - Contribuições relacionadas ao pensamento computacional relatadas

\begin{tabular}{llcc}
\hline \multicolumn{1}{c}{ CONTRIBUIÇÕES } & \multicolumn{1}{c}{ REFERÊNCIA DO ESTUDO } & QTD. & \% \\
\hline $\begin{array}{l}\text { O PC possibilita o desenvolvimento } \\
\text { de habilidades nos estudantes }\end{array}$ & $\begin{array}{l}\text { E18; E23; E29; E34; E39; E48; E49; E50; } \\
\text { E52; E53;E57; E59; E65; E70 }\end{array}$ & $32,39 \%$ \\
\hline $\begin{array}{l}\text { O PC pode ser viabilizado por meio } \\
\text { do uso de abordagens/estratégias } \\
\text { ou recursos/ferramentas }\end{array}$ & $\begin{array}{l}\text { E04; E05; E16; E21; E26; E36; E38; E39; } \\
\text { E48; E53;E56; E58; E59;E61 }\end{array}$ & 14 & $19,72 \%$ \\
\hline $\begin{array}{l}\text { O PC pode ser integrado a outras } \\
\text { matérias ou disciplinas }\end{array}$ & E31; E40; E41; E57; E58 & 5 & $7,04 \%$ \\
\hline
\end{tabular}

Fonte: Desenvolvida pelos autores.

Como pode ser percebido na Tabela 9, as contribuições relatadas pelos estudos são: o PC possibilita o desenvolvimento de habilidades nos estudantes, contribuição essa observada em $32,39 \%(n=23)$ dos estudos; o PC pode ser viabilizado por meio do uso de abordagens/estratégias ou recursos/ferramentas, contribuição presente em $19,72 \%$ ( $n=14)$; o PC pode ser integrado a outras matérias ou disciplinas, contribuição constatada em $7,04 \%(n=5)$.

Quanto às dificuldades relatadas, relacionadas ao pensamento computacional, os estudos E24, E28 e E60, ainda que de formas diferentes, evidenciam a necessidade de se ampliar investimentos e trabalhos para a difusão e emprego efetivo do pensamento computacional nos currículos, práticas docentes e nas atividades acadêmicas. Além dessa dificuldade, os estudos E23 e E45 apontam para a infraestrutura como outra dificuldade.

\section{CONSIDERAÇÕES FINAIS}

Neste artigo objetivou-se identificar e caracterizar estudos que tenham como foco o pensamento computacional, provenientes de pesquisas de Mestrado e Doutorado elaboradas a partir de programas de Pós-Graduação no Brasil. Para isso, foi realizada uma pesquisa de revisão sistemática de literatura, entre os anos de 2010 e 2019. Por meio da execução do protocolo de revisão sistemática, foram selecionados 71 estudos para compor a base desta revisão.

Por intermédio da análise desses estudos observou-se que os quantitativos de pesquisas produzidas com foco no desenvolvimento do pensamento computacional em programas de Pós-Graduação no Brasil, no período de 2010 e 2019 (QP1), evidenciam o interesse e a atualidade da temática, apresentando uma tendência de crescimento a partir do ano de 2015.

No que respeita às Unidades da Federação, instituições e programas de Pós-Graduação no Brasil que concentram essas pesquisas (QP2), verificou-se que os trabalhos concentram-se, principalmente, nos Estados de São Paulo, Rio Grande do Sul, Santa Catarina e Paraná, tendo como destaques as seguintes instituições: Universidade do Vale do Itajaí e Universidade Federal de Campina Grande, bem como os programas de Pós-Graduação em Ciência da Computação, Computação Aplicada, Educação, Matemática, Informática e Informática na Educação. 
No que diz respeito às abordagens desses estudos (QP3), salientam-se a promoção do pensamento computacional e a associação do PC à programação de computadores e à Matemática. Essas abordagens são desenvolvidas (QP4), geralmente, por meio da instituição de intervenção pedagógica ou cursos, desenvolvimento de ferramentas ou instrumentos, bem como a expansão de propostas de abordagem.

Referente às práticas educacionais associadas ou utilizadas nesses estudos (QP5), destacam-se o desenvolvimento ou utilização de jogos (digitais ou analógicos), a resolução de problemas, a aprendizagem baseada em atividades práticas, atividades desplugadas e lúdicas.

Em relação aos pressupostos teórico-pedagógicos associados ou utilizados nesses estudos (QP6), observa-se a notoriedade do embasamento pelo construcionismo e construtivismo. Por sua vez, no que compete aos recursos e/ou ferramentas utilizados nesses estudos (QP7), destacam-se o uso do Scratch e os kits de robótica educacional.

No que se refere aos níveis de ensino em que esses estudos são aplicados (QP8), observou-se que se concentram, majoritariamente, nos Ensinos Fundamental e Médio. Quanto às contribuições relacionadas ao pensamento computacional (QP9), relatadas pelos estudos, constata-se que, de um modo geral, o pensamento computacional possibilita o desenvolvimento de habilidades nos estudantes e pode ser viabilizado por meio do uso de abordagens/estratégias ou recursos/ferramentas. Também é possível ser integrado a outras matérias ou disciplinas.

Por outro lado, os estudos relatam como dificuldades a necessidade de se ampliar investimentos e trabalhos para a difusão e emprego efetivo do pensamento computacional nos currículos, além de práticas docentes e nas atividades acadêmicas, bem como na infraestrutura.

É válido destacar que esta revisão compartilha as limitações mais comuns do método sistemático, como a cobertura de pesquisa e possíveis vieses introduzidos durante a seleção dos estudos, extração de dados e análise. Ademais, no que se relaciona, especificamente, à análise de dados, registra-se, como uma limitação, o fato de algumas das questões de pesquisa elencadas requererem respostas que não sejam binárias ou objetivas. Convém assinalar, entretanto, que essas limitações foram abordadas seguindo as recomendações gerais para revisões sistemáticas.

Por fim, espera-se que esta revisão, por meio dos achados presentes neste texto, possa subsidiar outras pesquisas que abordem a temática, ao possibilitar o desenvolvimento e o aprofundamento das questões aqui abordadas.

\section{AGRADECIMENTOS}

O presente trabalho foi realizado com apoio do CNPq, Conselho Nacional de Desenvolvimento Científico e Tecnológico - Brasil (Bolsista do CNPq 310259/2020-7).

\section{REFERÊNCIAS}

AGBO, Friday Joseph et al. A Systematic Review of Computational Thinking Approach for Programming Education in Higher Education Institutions. In: Proceedings of the 19th Koli Calling International Conference on Computing Education Research. NY, USA: ACM, 2019. p. 1-10. Disponível em: https://doi. org/10.1145/3364510.3364521 
ARAUJO, Ana Liz; ANDRADE, Wilkerson; GUERRERO, Dalton. Um mapeamento sistemático sobre a avaliação do pensamento computacional no Brasil. In: WORKSHOPS DO CONGRESSO BRASILEIRO DE INFORMÁTICA NA EDUCAÇÃO, 2016. Anais [...]. 2016. p. 1147. Disponível em: https://doi.org/10.5753/cbie.wcbie.2016.1147

AVILA, Christiano et al. Metodologias de avaliação do pensamento computacional: uma revisão sistemática. In: SIMPÓSIO BRASILEIRO DE INFORMÁTICA NA EDUCAÇÃO (SBIE 2017), 28., 2017. Anais [...]. 2017. p. 113. Disponível em: https://doi.org/10.5753/cbie.sbie.2017.113

BARBOSA, Luciana. A inserção do pensamento computacional na Base Nacional Comum Curricular: reflexões acerca das implicações para a formação inicial dos professores de matemática. In: WORKSHOP DE INFORMÁTICA NA ESCOLA (WIE 2019), 25., 2019. Anais [...]. p. 889-898. Disponível em: https://doi. org/10.5753/cbie.wie.2019.889

BARCELOS, Thiago et al. Relações entre o pensamento computacional e a matemática: uma revisão sistemática da literatura. In: WORKSHOPS CONGRESSO BRASILEIRO DE INFORMÁTICA NA EDUCAÇÃO (CBIE 2015), 4., 2015. Anais [...]. 2015. p. 1.369. Disponível em: https://doi.org/10.5753/cbie.wcbie.2015.1369 BARCELOS, Thiago S. et al. Mathematics learning through computational thinking activities: A systematic literature review. Journal of Universal Computer Science, v. 24, n. 7, p. 815-845, 2018.

BOMBASAR, James et al. Ferramentas para o ensino-aprendizagem do pensamento computacional: onde está Alan Turing? In: SIMPÓSIO BRASILEIRO DE INFORMÁTICA NA EDUCAÇÃO (SBIE 2015), 26., 2015. Anais [...]. 2015. p. 81. Disponível em: https://doi.org/10.5753/cbie.sbie.2015.81

BRASIL. Base Nacional Comum Curricular: educação é a base. MEC, 2018. Disponível em: http://basenacionalcomum.mec.gov.br/images/BNCC_EI_EF_110518_versaofinal_site.pdf. Acesso em: 13 nov. 2020. BUITRAGO FLÓREZ, Francisco et al. Changing a Generation's Way of Thinking: Teaching Computational Thinking Through Programming. Review of Educational Research, v. 87, n. 4, p. 834-860, 2017. Disponível em: https://doi.org/10.3102/0034654317710096

CARVALHO, Joethe; NETTO, José Francisco; ALMEIDA, Thais. Revisão sistemática de literatura sobre pensamento computacional por meio de objetos de aprendizagem. In: SIMPÓSIO BRASILEIRO DE INFORMÁTICA NA EDUCAÇÃO (SBIE 2017), 28., 2017. Anais [...]. 2017. p. 223. Disponível em: https://doi.org/10.5753/ cbie.sbie.2017.223

CHAO, Po-Yao. Exploring students' computational practice, design and performance of problem-solving through a visual programming environment. Computers \& Education, v. 95, p. 202-215, 2016. Disponível em: https://doi.org/10.1016/j.compedu.2016.01.010

DA SILVA, Emanuel Oliveira; FALCÃO, Taciana Pontual. O pensamento computacional no Ensino Superior e seu impacto na aprendizagem de programação. In: WORKSHOP SOBRE EDUCAÇÃO EM COMPUTAÇÃO (WEI 2020), 2020. Anais [...]. 2020. p. 171-175. Disponível em: https://doi.org/10.5753/wei.2020.11152

DYBÅ, Tore; DINGS $\varnothing$ YR, Torgeir. Empirical studies of agile software development: A systematic review. Information and Software Technology, v. 50, n. 9-10, p. 833-859, 2008. Disponível em: https://doi.org/10.1016/j.infsof.2008.01.006

EINHORN, Susan. Microworlds, computational thinking, and 21st century learning. LCSI White Paper, p. 1-10, 2011. Disponível em: https://el.media.mit.edu/logo-foundation/resources/papers/pdf/computational_thinking.pdf. Acesso em: 10 nov. 2020.

FARIAS, Adelito; ANDRADE, Wilkerson; ALENCAR, Rayana. Pensamento computacional em sala de aula: desafios, possibilidades e a formação docente. In: WORKSHOPS DO CONGRESSO BRASILEIRO DE INFORMÁTICA NA EDUCAÇÃO (CBIE 2015), 4., 2015. Anais [...]. 2015. p. 1226. Disponível em: https://doi. org/10.5753/cbie.wcbie.2015.1226

FRANÇA, Rozelma; TEDESCO, Patrícia. Desafios e oportunidades ao ensino do pensamento computacional na educação básica no Brasil. In: WORKSHOPS DO CONGRESSO BRASILEIRO DE INFORMÁTICA NA EDUCAÇÃO (CBIE 2015), 4., 2015. Anais [...]. 2015. p. 1.464. Disponível em: https://doi.org/10.5753/cbie.wcbie.2015.1464

FRANÇA, Rozelma; TEDESCO, Patrícia. Pensamento computacional: panorama dos grupos de pesquisa no Brasil. In: SIMPÓSIO BRASILEIRO DE INFORMÁTICA NA EDUCAÇÃO (SBIE 2019), 30., 2019. Anais [...]. 2019. p. 409. Disponível em: https://doi.org/10.5753/cbie.sbie.2019.409

GALVÃO, Taís Freire; PEREIRA, Mauricio Gomes. Avaliação da qualidade da evidência de revisões sistemáticas. Epidemiologia e Serviços de Saúde, v. 24, n. 1, p. 775-778, 2015. Disponível em: https://doi. org/10.5123/S1679-49742015000100019

GROVER, Shuchi; PEA, Roy. Computational Thinking in K-12. Educational Researcher, v. 42, n. 1, p. 38-43, 2013. Disponível em: https://doi.org/10.3102/0013189X12463051 
HICKMOTT, Daniel; PRIETO-RODRIGUEZ, Elena; HOLMES, Kathryn. A Scoping Review of Studies on Computational Thinking in K-12 Mathematics Classrooms. Digital Experiences in Mathematics Education, v. 4, $\mathrm{n}$. 1, p. 48-69, 2018. Disponível em: https://doi.org/10.1007/s40751-017-0038-8

HSU, Ting-Chia; CHANG, Shao-Chen; HUNG, Yu-Ting. How to learn and how to teach computational thinking: Suggestions based on a review of the literature. Computers \& Education, v. 126, p. 296-310, 2018. Disponível em: https://doi.org/10.1016/j.compedu.2018.07.004

KITCHENHAM, Barbara A. Systematic review in software engineering. Association for Computing Machinery (ACM), 2012. p. 1. Disponível em: https://doi.org/10.1145/2372233.2372235

KONG, Siu Cheung; CHIU, Ming Ming; LAI, Ming. A study of primary school students' interest, collaboration attitude, and programming empowerment in computational thinking education. Computers \& Education, v. 127, p. 178-189, 2018. Disponível em: https://doi.org/10.1016/j.compedu.2018.08.026

ORTIZ, Julia Dos S. B.; PEREIRA, Roberto. Um Mapeamento Sistemático Sobre as Iniciativas para Promover o Pensamento Computacional. In: SIMPÓSIO BRASILEIRO DE INFORMÁTICA NA EDUCAÇÃO (SBIE 2018), 29., 2018. Anais [...]. 2018. p. 1.093. Disponível em: https://doi.org/10.5753/cbie.sbie.2018.1093

PAPERT, Seymour A. Mindstorms: Children, computers, and powerful ideas. NY, USA: Basic Books, 1980.

PASQUAL JÚNIOR, Paulo Antonio; OLIVEIRA, Simone de. Pensamento Computacional: uma proposta de oficina para a formação de professores. Renote, v. 17, n. 1, p. 62-71, 2019. Disponível em: https://doi. org/10.22456/1679-1916.95707

POULAKIS, Emmanouil; POLITIS, Panagiotis. Teaching Computational Thinking Unplugged: A Review of Tools and Methodologies. In: Handbook of Research on Tools for Teaching Computational Thinking in P-12 Education. Hershey, 2020. p. 200-236. Disponível em: https://doi.org/10.4018/978-1-7998-4576-8.ch009 RODRIGUES, Sebastião; ARANHA, Eduardo; SILVA, Thiago Reis. Computação desplugada no ensino de programação: uma revisão sistemática da literatura. In: SIMPÓSIO BRASILEIRO DE INFORMÁTICA NA EDUCAÇÃO (SBIE 2018), 29., 2018. Anais [...]. 2018. p. 417. Disponível em: https://doi.org/10.5753/cbie. sbie.2018.417

ROMÁN-GONZÁLEZ, Marcos; MORENO-LEÓN, Jesús; ROBLES, Gregorio. Complementary Tools for Computational Thinking Assessment. In: INTERNATIONAL CONFERENCE ON COMPUTATIONAL THINKING EDUCATION (CTE 2017), Proceedings [...]. 2017. p. 154-159.

ROSA, Valdir; COELHO NETO, João. Design Thinking e o pensamento computacional e suas articulações para o ensino de robótica educacional: uma revisão. Research, Society and Development, v. 9, n. 10, 2020. Disponível em: https://doi.org/10.33448/rsd-v9i10.9019

SELBY, Cynthia C. Relationships: Computational thinking, pedagogy of programming, and Bloom's Taxonomy. In: WORKSHOP IN PRIMARY AND SECONDARY COMPUTING EDUCATION ON ZZZ - WIPSCE '15. NY, USA, 2015. Proceedings [...].NY, USA: ACM Press, 2015. p. 80-87. Disponível em: https://doi. org/10.1145/2818314.2818315

SILVA, Kennedy dos Santos Silva; PEREIRA, Nícolas Pierim; ODAKURA, Valguima. Mapeamento sistemático: estratégias para o ensino-aprendizagem do pensamento computacional no Brasil. Nuevas Ideas en Informática Educativa, v. 14, p. 319-329, 2018.

SILVA, Vladimir; SILVA, Luis Lima da; FRANÇA, Rozelma. Pensamento computacional na formação de professores: experiências e desafios encontrados no ensino da computação em escolas públicas. In: WORKSHOP DE INFORMÁTICA NA ESCOLA (WIE 2017), 23., 2017. Anais [...]. 2017. p. 805. Disponível em: https://doi.org/10.5753/cbie.wie.2017.805

TANG, Xiaodan et al. Assessing computational thinking: A systematic review of empirical studies. Computers \& Education, v. 148, 2020. Disponível em: https://doi.org/10.1016/j.compedu.2019.103798

WING, Jeannette M. Computational thinking. Communications of the ACM, v. 49, n. 3, p. 33-35, 2006. Disponível em: https://doi.org/10.1145/1118178.1118215

YADAV, Aman et al. Expanding computer science education in schools: understanding teacher experiences and challenges. Computer Science Education, v. 26, n. 4, p. 235-254, 2017. Disponível em: https://doi. org/10.1080/08993408.2016.1257418

YADAV, Aman; HONG, Hai; STEPHENSON, Chris. Computational Thinking for All: Pedagogical Approaches to Embedding 21st Century Problem Solving in K-12 Classrooms. TechTrends, v. 60, n. 6, p. 565-568, 2016. Disponível em: https://doi.org/10.1007/s11528-016-0087-7

YADAV, Aman; STEPHENSON, Chris; HONG, Hai. Computational Thinking for Teacher Education. Communications of the ACM, v. 60, n. 4, p. 55-62, 2017. Disponível em: https://doi.org/10.1145/2994591 
YEN, Ching-Zon; WU, Ping-Huang; LIN, Ching-Fang. Analysis of Experts' and Novices' Thinking Process in Program Debugging. In: Communications in Computer and Information Science, v. 302 CCIS, p. 122-134, 2012. Disponível em: https://doi.org/10.1007/978-3-642-31398-1_12

ZANETTI, Humberto; BORGES, Marcos; RICARTE, Ivan. Pensamento computacional no ensino de programação: uma revisão sistemática da literatura brasileira. In: SIMPÓSIO BRASILEIRO DE INFORMÁTICA NA EDUCAÇÃO (SBIE 2016), 27., 2016. Anais [...]. 2016. p. 21. Disponível em: https://doi.org/10.5753/cbie. sbie.2016.21

ZHANG, LeChen; NOURI, Jalal. A systematic review of learning computational thinking through Scratch in K-9. Computers \& Education, v. 141, 2019. Disponível em: https://doi.org/10.1016/j.compedu.2019.103607 


\section{APÊNDICE A - ESTUDOS SELECIONADOS 3}

[E01] ACHUTTI, Camila Fernandez. Framework cascas de árvore: rearranjos nas competências e mentalidade para a alfabetização digital e tecnológica em tempos de taxas exponenciais de mudança. 2017. Dissertação (Mestrado em Ciências da Computação) - Universidade de São Paulo, Instituto de Matemática e Estatística, São Paulo, 2017.

[E02] ALVES, Nathalia da Cruz. CodeMaster: um modelo de avaliação do pensamento computacional na educação básica através da análise de código de linguagem de programação visual. 2019. Dissertação (Mestrado em Ciência da Computação) - Universidade Federal de Santa Catarina, Florianópolis, 2019.

[E03] ARAUJO, Ana Liz Souto Oliveira de. Quantifying computational thinking abilities. 2019. Tese (Doutorado em Ciência da Computação) - Universidade Federal de Campina Grande, Centro de Engenharia Elétrica e Informática, Campina Grande, 2019.

[E04] BAGESTAN, Diego Berti. Ressignificando a lógica de programação: a utilização do software Scratch em um curso técnico em informática. 2018. Dissertação (Mestrado) - Universidade do Vale do Taquari, Curso de Ensino de Ciências Exatas, Lajeado, 2018.

[E05] BARBOSA, Lara Martins. Aspectos do pensamento computacional na construção de fractais com o software GeoGebra. 2019. Dissertação (Mestrado em Educação Matemática) - Universidade Estadual Paulista, Instituto de Geociências e Ciências Exatas, Rio Claro, 2019.

[E06] BARCAROLI, Velcir. Plataforma interativa de aprendizagem de programação voltada à disseminação do pensamento computacional utilizando robótica remota. 2017. Dissertação (Mestrado em Computação Aplicada) - Universidade de Passo Fundo, Passo Fundo, 2017.

[E07] BARCELOS, Thiago Schumacher. Relações entre o pensamento computacional e a matemática em atividades didáticas de construção de jogos digitais. 2014. Tese (Doutorado em Ensino de Ciências e Matemática) - Universidade Cruzeiro do Sul, São Paulo, 2014.

[E08] BENARRÓS, Cynara Rodrigues. Sequência didática para o ensino-aprendizagem de informática no curso de assistente administrativo da educação profissional. 2017. Dissertação (Mestrado Profissional em Ensino Tecnológico) - Instituto Federal do Amazonas, Manaus, 2017.

[E09] BOMBASAR, James Roberto. Computability Game - um jogo de lógica inspirado na máquina de turing para apoio ao desenvolvimento do pensamento computacional. 2017. Dissertação (Mestrado em Computação Aplicada) - Universidade do Vale do Itajaí, Itajaí, 2017.

[E10] BOUCINHA, Rafael Marimon. Aprendizagem do pensamento computacional e desenvolvimento do raciocínio. 2017. Tese (Doutorado em Informática na Educação) - Universidade Federal do Rio Grande do Sul, Centro de Estudos Interdisciplinares em Novas Tecnologias na Educação, Porto Alegre, 2017.

[E11] BRACKMANN, Christian PuhImann. Desenvolvimento do pensamento computacional através de atividades desplugadas na educação básica. 2017. Tese (Doutorado em Informática na Educação) - Universidade Federal do Rio Grande do Sul, Centro Interdisciplinar de Novas Tecnologias na Educação, Porto Alegre, 2017.

[E12] BREMM, Cristiane Inês. Mediação do pensamento computacional e programação no processo de interação das crianças na educação infantil. 2018. Dissertação (Mestrado Profissional em Tecnologias Educacionais em Rede) - Universidade Federal de Santa Maria, Centro de Educação, Santa Maria, 2018.

[E13] BRESSAN, Manuelle Lopes Quintas. Scratch! um estudo de caso. 2016. Dissertação (Mestrado em Tecnologia e Sociedade) - Universidade Tecnológica Federal do Paraná, Curitiba, 2016.

[E14] CÂMARA, Fábio Sampaio dos Santos. Desenvolvimento de habilidades matemáticas com a inclusão do pensamento computacional nas escolas de Ensino Fundamental. 2019. Dissertação (Mestrado Profissional em Inovação em Tecnologias Educacionais) - Universidade Federal do Rio Grande do Norte, Instituto Metrópole Digital, Natal, 2019.

[E15] CAMPOS, Luciana Xavier de. Pensamento computacional, Scratch e resolução de problemas: uma pesquisa intervenção com alunos do 70 ano do Ensino Fundamental. 2018. Dissertação (Mestrado Profissional em Computação Aplicada) - Universidade Estadual do Ceará, Centro de Ciências e Tecnologia, Fortaleza, 2018.

Os dados relativos à pesquisa podem ser consultados por meio do seguinte link: bit.ly/Artigo-PC2021-RCE 
[E16] CASTILHO, Maria Inês. Hiperobjetos da robótica educacional como ferramentas para o desenvolvimento da abstração reflexionante e do pensamento computacional. 2018. Tese (Doutorado em Informática na Educação) - Universidade Federal do Rio Grande do Sul, Centro Interdisciplinar de Novas Tecnologias na Educação, Porto Alegre, 2018.

[E17] COSTA, Erick John Fidelis. Pensamento computacional na educação básica: uma abordagem para estimular a capacidade de resolução de problemas na matemática. 2017. Dissertação (Mestrado em Ciência da Computação) - Universidade Federal de Campina Grande, Centro de Engenharia Elétrica e Informática, Paraíba, 2017.

[E18] COUTO, Natalia Ellery Ribeiro. Impacto da implantação de uma disciplina de pensamento computacional em estudantes: um estudo de caso. 2019. Dissertação (Mestrado em Computação Aplicada) - Universidade do Vale do Itajaí, Itajaí, 2019.

[E19] CROVADOR, Alvaro. O Uso do arduino em sala de aula no experimento do pêndulo simples de Galileu Galilei. 2019. Dissertação (Mestrado Profissional em Educação e Novas Tecnologias) - Centro Universitário Internacional Uninter, Curitiba, 2019.

[E20] DUARTE, Ailton Souza. O ensino do pensamento computacional na educação profissional de nível médio no Ifes - Campus Colatina. 2018. Dissertação (Mestrado em Ciências em Educação Agrícola) - Universidade Federal Rural do Rio de Janeiro, Instituto de Agronomia, Seropédica, 2018.

[E21] EGIDO, Sidnéia Valero. Educação matemática e desenvolvimento do pensamento computacional no 3o ano do Ensino Fundamental: crianças programando jogos com Scratch. 2018. Dissertação (Mestrado em Ciências e Matemática) - Universidade Federal do Paraná, Curitiba, 2018.

[E22] ELOY, Adelmo Antonio da Silva. Contribuições para aplicação de learning analytics no apoio à avaliação em atividades de introdução à programação com Scratch. 2019. Dissertação (Mestrado em Sistemas Eletrônicos) - Universidade de São Paulo, Escola Politécnica, São Paulo, 2019.

[E23] EVARISTO, Ingrid Santella. O pensamento computacional no processo de aprendizagem da matemática nos anos finais do Ensino Fundamental. 2019. Dissertação (Mestrado em Gestão e Práticas Educacionais) - Universidade Nove de Julho, São Paulo, 2019.

[E24] FERNANDES, Hugo Batista. Pensamento computacional: uma proposta de curso de extensão on-line para professores que lecionam matemática nos anos iniciais do ensino fundamental. 2018. Dissertação (Mestrado em Ensino de Ciências e Matemática) - Universidade Cruzeiro do Sul, São Paulo, 2018.

[E25] FERRI, Juliana. Ensino de linguagem de programação na educação básica: uma proposta de sequência didática para desenvolver o pensamento computacional. 2017. Dissertação (Mestrado Profissional em Ensino) - Universidade Estadual do Norte do Paraná, Cornélio Procópio, 2017.

[E26] FORQUESATO, Luís Eduardo Thibes. Usando um jogo para ensinar pensamento computacional e avaliar o aprendizado. 2018. Dissertação (Mestrado) - Universidade Estadual de Campinas, Instituto de Computação, Campinas, SP, 2018.

[E27] FRANÇA, Rozelma Soares de. Um modelo para a aprendizagem do pensamento computacional aliado à autorregulação. 2015. Dissertação (Mestrado em Ciência da Computação) - Universidade Federal de Pernambuco, Centro de Informática, Recife, 2015.

[E28] GERALDES, Wendell Bent. O pensamento computacional no ensino profissional e tecnológico. 2017. Dissertação (Mestrado em Gestão do Conhecimento e da Tecnologia da Informação) - Universidade Católica de Brasília, Brasília, 2017.

[E29] GLIZT, Fabiana Rodrigues de Oliveira. O pensamento computacional nos anos iniciais do Ensino Fundamental. 2017. Dissertação (Mestrado em Ensino de Ciência e Tecnologia) - Universidade Tecnológica Federal do Paraná, Ponta Grossa, 2017.

[E30] GONCALVES, Filipe Augusto. Um instrumento para o diagnóstico do pensamento computacional. 2015. Dissertação (Mestrado em Computação Aplicada) - Universidade do Vale do Itajaí, Itajaí, 2015.

[E31] GREFF, Guaraci Vargas. Pensamento computacional na educação básica: uma proposta interdisciplinar de mobilização para o processo ensino-aprendizagem da língua portuguesa. 2019. Dissertação (Mestrado Profissional em Informática na Educação) - Instituto Federal de Educação, Ciência e Tecnologia do Rio Grande do Sul, Campus Porto Alegre, 2019.

[E32] KAMINSKI, Márcia Regina. Análise das práticas de informática na educação da Escola Municipal Aloys João Mann - Cascavel/PR. 2018. Dissertação (Mestrado em Ensino) - Universidade Estadual do Oeste do Paraná, Foz do Iguaçu, 2018.

[E33] LIMA, William Vieira de. Percepção ambiental e desenvolvimento Scratch: uso da água no pulsar do Rio Juruá - Eirunepé - Amazonas. 2018. Dissertação (Mestrado em Ensino das Ciências Ambientais) Universidade Federal do Amazonas, Manaus, 2018. 
[E34] LUMMERTZ, Ramon dos Santos. As potencialidades do uso do software Scratch para a construção da literacia digital. 2016. Dissertação (Mestrado em Ensino de Ciências e Matemática) - Universidade Luterana do Brasil, Canoas, 2016.

[E35] LUQUE CARBAJAL, Marleny. Design e desenvolvimento de um ambiente de programação tangível de baixo custo para crianças. 2016. Dissertação (Mestrado em Ciência da Computação) - Instituto de Computação, Universidade Estadual de Campinas, Campinas, 2016.

[E36] MARQUES, Samanta Ghisleni. Implicação dos pilares do pensamento computacional na resolução de problemas na escola. 2019. Dissertação (Mestrado em Educação) - Universidade de Santa Cruz do Sul, Santa Cruz do Sul, 2019.

[E37] MARTINELLI, Suéllen Rodolfo. MultiTACT: uma abordagem para a construção de atividades de ensino multidisciplinares para estimular o Pensamento Computacional no Ensino Fundamental I. 2019. Dissertação (Mestrado em Ciência da Computação) - Centro de Ciências em Gestão e Tecnologia, Universidade Federal de São Carlos, Sorocaba, 2019.

[E38] MARTINS, Paulo Eduardo. Desenvolvimento de um plug-in do Portugol Studio para criação de Apps. 2019. Dissertação (Mestrado em Computação Aplicada) - Universidade do Vale do Itajaí, Itajaí, 2019.

[E39] MEIRA, Ricardo Radaelli. Pensamento computacional na educação básica: uma proposta metodológica com jogos e atividades lúdicas. 2017. Dissertação (Mestrado Profissional em Tecnologias Educacionais em Rede) - Universidade Federal de Santa Maria, Centro de Educação, Santa Maria, 2017.

[E40] MESTRE, Palloma Alencar Alves. O uso do pensamento computacional como estratégia para resolução de problemas matemáticos. 2017. Dissertação (Mestrado em Ciência da Computação) - Universidade Federal de Campina Grande, Centro de Engenharia Elétrica e Informática, Paraíba, 2017.

[E41] NASCIMENTO, Carlos Alexandre. Integração entre ciência da computação e língua portuguesa por meio de habilidades do pensamento computacional. 2018. Dissertação (Mestrado em Ciência da Computação) - Universidade Federal da Bahia, Instituto de Matemática e Estatística, Salvador, 2018.

[E42] NASCIMENTO, Renata Melo. A matemática e VisuAlg: lógica de programação no Ensino Médio. 2019. Dissertação (Mestrado Profissional em Matemática em Rede Nacional) - Universidade Estadual de Santa Cruz, Ilhéus, 2019.

[E43] NUNES, Cinthia Batista. Introdução à computação: uma proposta para o ensino básico. 2013. Dissertação (Mestrado Profissional em Matemática em Rede Nacional) - Universidade Estadual do Sudoeste da Bahia, Vitória da Conquista, 2013.

[E44] ORTIZ, Julia dos Santos Bathke. Pensamento computacional e educação de jovens e adultos: na direção de um modelo socialmente consciente. 2019. Dissertação (Mestrado em Informática) - Setor de Ciências Exatas, Universidade Federal do Paraná, Curitiba, 2019.

[E45] PASQUAL JÚNIOR, Paulo Antonio. Pensamento computacional e formação de professores: uma análise a partir da plataforma Code.org. 2018. Dissertação (Mestrado em Educação) - Universidade de Caxias do Sul, Caxias do Sul, 2018.

[E46] PAUCAR CURASMA, Herminio. Uma ferramenta para a introdução à programação e pensamento computacional com motivação usando realidade virtual. 2017. Dissertação (Mestrado em Informática) - Pontifícia Universidade Católica do Rio de Janeiro, Departamento de Informática, Rio de Janeiro, 2017.

[E47] PEREIRA, João Pedro de Lima. Programação e pensamento computacional no 8 e 9o ano do Ensino Fundamental: um estudo de caso. 2019. Dissertação (Mestrado Profissional em Matemática) - Universidade de Brasília, Brasília, 2019.

[E48] POLONI, Leonardo. Aprendizagem de programação mediada por uma linguagem visual: possibilidade de desenvolvimento do pensamento computacional. 2018. Dissertação (Mestrado em Educação) - Universidade de Caxias do Sul, Caxias do Sul, 2018.

[E49] QUEIROZ, Rubens Lacerda. DuinoBlokcks4Kids: utilizando Tecnologia Livre e materiais de baixo custo para o exercício do Pensamento Computacional no Ensino Fundamental I por meio do aprendizado de programação aliado à Robótica Educacional. 2017. Dissertação (Mestrado em Informática) - Universidade Federal do Rio de Janeiro, Instituto de Matemática, Instituto Tércio Pacitti de Aplicações e Pesquisas Computacionais, Rio de Janeiro, 2017.

[E50] RIBOLDI, Sandra Mara Oselame. A linguagem de programação Scratch e o ensino de funções: uma possibilidade. 2019. Dissertação (Mestrado Profissional em Matemática em Rede Nacional) - Universidade Federal da Fronteira Sul, Chapecó, 2019.

[E51] ROCHA, José Rafael Moraes Garcia da. Developing programming skills on digital native children through the interaction with smart devices. 2016. Dissertação (Mestrado em Ciência da Computação) Universidade Federal de Pernambuco, Centro de Informática, Recife, 2016. 
[E52] RODRIGUES, Rivanilson da Silva. Um estudo sobre os efeitos do pensamento computacional na educação. 2016. Dissertação (Mestrado em Ciência da Computação) - Universidade Federal de Campina Grande, Centro de Engenharia Elétrica e Informática, Paraíba, 2016.

[E53] ROSARIO, Tatiane Aparecida Martins do. As aprendizagens com o uso do brinquedo de programar: um estudo com crianças de cinco e seis anos de idade de uma instituição de Educação Infantil. 2017. Dissertação (Mestrado em Educação) - Universidade do Vale do Itajaí, Itajaí, 2017.

[E54] SANTANA, Andre Luiz Maciel. Análise do processo metodológico de montagem de um brinquedo de programar. 2015. Dissertação (Mestrado em Computação Aplicada) - Universidade do Vale do Itajaí, Itajaí, 2015.

[E55] SANTANNA, Hugo Cristo. Ação, computação, representação: um estudo psicogenético sobre o desenvolvimento do pensamento computacional. 2014. Tese (Doutorado em Psicologia) - Universidade Federal do Espírito Santo, Vitória, 2014.

[E56] SANTIN, Mateus Madail. Desenvolvimento do pensamento computacional através da robótica: fluidez digital no ensino fundamental. 2014. Tese (Doutorado em Educação em Ciências) - Universidade Federal do Rio Grande, Rio Grande, 2014.

[E57] SANTOS, Cícero Gonçalves dos. Estratégias para implantação e avaliação de um método educacional desplugado com histórias em quadrinhos para o ensino e aprendizagem associados ao desenvolvimento do pensamento computacional com alunos do Ensino Fundamental. 2019. Dissertação (Mestrado em Ciência da Computação) - Universidade Federal de Sergipe, São Cristóvão, 2019.

[E58] SANTOS, Gilson Pedroso dos. Educação e tecnologia no interior da Amazônia: o pensamento computacional e as tecnologias da informação e comunicação como auxílio em processo de ensino-aprendizagem. 2018. Dissertação (Mestrado em Educação) - Universidade Federal do Oeste do Pará, Instituto de Ciências da Educação, Santarém, 2018.

[E59] SILVA, Eliel Constantino da. Pensamento computacional e a formação de conceitos matemáticos nos anos finais do Ensino Fundamental: uma possibilidade com kits de robótica. 2018. Dissertação (Mestrado em Educação Matemática) - Universidade Estadual Paulista, Instituto de Geociências e Ciências Exatas, Rio Claro, 2018.

[E60] SILVA, Hélio Moreira da. Pensamento computacional: desenvolvimento do material pedagógico para o ensino de programação de games 2D na educação básica auxiliado pelo design de interação. 2019. Dissertação (Mestrado Profissional em Design, Tecnologia e Inovação) - Centro Universitário Teresa D’Ávila, Lorena, 2019.

[E61] SILVA, Jéssica Laísa Dias da. Game Design de jogos digitais de pensamento computacional inspirados no instrumento de avaliação Bebras Challenge. 2018. Dissertação (Mestrado em Sistemas e Computação) - Universidade Federal do Rio Grande do Norte, Centro de Ciências Exatas e da Terra, Natal, 2018.

[E62] SILVA, Leonardo Cintra Lopes da. A relação do pensamento computacional com o ensino de matemática na educação básica. 2019. Dissertação (Mestrado em Matemática em Rede Nacional) - Universidade Estadual Paulista, Faculdade de Ciências e Tecnologia, Presidente Prudente, 2019.

[E63] SILVA, Mariana Cardoso da. Robótica Educacional Livre: um relato de prática no Ensino Fundamental. 2017. Dissertação (Mestrado em Educação: Currículo) - Pontifícia Universidade Católica de São Paulo, São Paulo, 2017.

[E64] SOUZA, Alexandra Aparecida de. Framework baseado em ciência de dados e engenharia de software para analisar a evolução de habilidades e competências do pensamento computacional. 2019. Tese (Doutorado em Engenharia Elétrica e Computação) - Universidade Presbiteriana Mackenzie, São Paulo, 2019.

[E65] SOUZA, Isabelle Maria Lima de. Aplicações da robótica educacional para o desenvolvimento do pensamento computacional no contexto da educação básica. 2019. Dissertação (Mestrado em Ciência da Computação) - Universidade Federal de Campina Grande, Centro de Engenharia Elétrica e Informática, Paraíba, 2019.

[E66] SOUZA, Leandro Delgado de. Instituto de hackers: o pensamento computacional aplicado ao ensino técnico integrado ao ensino médio. 2019. Dissertação (Mestrado em Educação Profissional e Tecnológica) - Instituto Federal do Paraná, Curitiba, 2019.

[E67] STELLA, Ana Lucia. Utilizando o pensamento computacional e a computação criativa no ensino da linguagem de programação Scratch para alunos do ensino fundamental. 2016. Dissertação (Mestrado em Tecnologia) - Universidade Estadual de Campinas, Faculdade de Tecnologia, Limeira, 2016. 
[E68] TASSANO, Débora Paola Rodríguez. Um olhar sobre teorias cognitivas: promovendo o aprendizado de lógica e programação. 2016. Dissertação (Mestrado Profissional em Educação e Tecnologia) - Instituto Federal Sul-Rio-Grandense, Pelotas, 2016.

[E69] VIDAL, José Augusto Mendes. Um estudo exploratório sobre o uso da robótica educacional como ferramenta de apoio ao ensino-aprendizagem de lógica de programação para alunos da rede pública do Ensino Médio. 2019. Dissertação (Mestrado em Informática) - Universidade Federal do Rio de Janeiro, Instituto de Matemática, Instituto Tércio Pacitti de Aplicações e Pesquisas Computacionais, Rio de Janeiro, 2019.

[E70] VIEIRA, Marli Fatima Vick. Pensamento computacional com enfoque construcionista no desenvolvimento de diferentes aprendizagens. 2018. Tese (Doutorado em Educação) - Universidade do Vale do Itajaí, Itajaí, 2018.

[E71] ZANCHETT, Guilherme Alexandre. Framework para auxiliar o desenvolvimento de jogos que abordem o pensamento computacional. 2016. Dissertação (Mestrado em Computação Aplicada) - Universidade do Vale do Itajaí, Itajaí, 2016. 